\title{
Total Serum Bile Acid as a Potential Marker for the Diagnosis of Cholangiocarcinoma without Jaundice
}

\author{
Sutthikan Sombattheera ${ }^{1}$, Tanakorn Proungvitaya ${ }^{1,2}$, Temduang Limpaiboon ${ }^{1,2}$, \\ Sopit Wongkham ${ }^{2}$, Chaisiri Wongkham ${ }^{2}$, Vor Luvira ${ }^{2}$, Siriporn Proungvitaya ${ }^{1,2 *}$
}

\begin{abstract}
Diagnosis of cholangiocarcinoma (CCA) is difficult when patients do not show jaundice. The aim of this study was to examine the feasibility of using the total serum bile acid (TSBA) level as an aid for the diagnosis of CCA in patients without jaundice. For this purpose, TSBA of the following groups were measured using a Beckman Synchron CX4 clinical chemistry analyzer: 60 cases of CCA with total serum bilirubin $\leq 2 \mathrm{mg} / \mathrm{dL}$ (low total bilirubin group, LTB); 32 cases of CCA with total serum bilirubin $>2 \mathrm{mg} / \mathrm{dL}$ (high total bilirubin group, HTB); and 115 healthy controls. Liver function parameters such as serum cholesterol, albumin, alanine aminotransferase (ALT), aspartate aminotransferase (AST) and alkaline phosphatase (ALP) were also examined. The results showed that the TSBA of both LTB and HTB groups of the CCA patients were significantly higher than that of the healthy controls. Also, significant correlation was observed between TSBA and total bilirubin levels in the HTB group of CCA patients. However, no such correlation was seen in the LTB group. The cut-off value of TSBA was determined for the LTB group of CCA patients using the receiver operating characteristic curve analysis, and it was $6.05 \mu \mathrm{mol} / \mathrm{L}$ with the sensitivity and specificity of $46.7 \%$ and $84.4 \%$, respectively. In addition, the ALP level was correlated well with the TSBA level and ALP in HTB group was significantly higher than that of LTB group. Moreover, the combination of high TSBA and high ALP levels gave higher specificity up to $\mathbf{9 7 . 4 \%}$. TSBA might be useful for the diagnosis of CCA patients without jaundice.
\end{abstract}

Keywords: Cholangiocarcinoma - total serum bile acid - jaundice-free status - diagnosis

Asian Pac J Cancer Prev, 16 (4), 1367-1370

\section{Introduction}

Cholangiocarcinoma (CCA) is a cancer of biliary epithelial cells. The prevalence of CCA shows a wide geographic variability, with the highest rates in Asia, especially Southeast Asia (Blechacz and Gores, 2008). The incidence of intrahepatic CCA is highest in the Northeast Thailand, especially in Khon Kaen province (44.3/100,000 in men and 17.6/100,000 in women) (Wiangnon et al., 2012), because of the high prevalence of carcinogenic liver fluke, Opisthorchis viverrini, infection (Sripa et al., 2007; Sripa et al., 2011).

CCA patients are asymptomatic in an early stage, but often become symptomatic later in relation to obstructive jaundice, for example, clay-colored stools, bilirubinuria, bilirubinemia, pruritus, dyspepsia, weight loss, and abdominal pain (Braconi and Patel, 2010; Zografos et al., 2011; Bhudhisawasdi et al., 2012). Thus, CCA patients can be divided into two groups, those with obstructive jaundice and without jaundice, which can be distinguished by high and low levels of total bilirubin level in serum. Diagnosis of CCA without jaundice is often delayed and only made at the late stage. Interest has, therefore, been focused on serum markers (Wongkham and Silsirivanit, 2012).

Alterations in serum bile acid metabolism are considered as the reflection of liver function (Heaton, 1979). Since bile acid metabolism mostly occurs in hepatocytes, accumulation of bile acids will occur if there is impairment in hepatocyte uptake, synthesis or secretion (Webster et al., 2002). The elevation of total serum bile acids (TSBA) was reported in patients with hepatocellular carcinoma and CCA (Changbumrung et al. 1990). In both patients groups, 86 and $92 \%$ of the patients showed higher level of serum total bilirubin than the upper limit of the normal controls.

The aim of this study is to evaluate whether the total serum bile acid (TSBA) can be used for the diagnostic marker for CCA patients without jaundice. For this purpose, we divided CCA patients into two entities, those with total serum bilirubin $\leq 2 \mathrm{mg} / \mathrm{dL}$ (LTB) and those with total serum bilirubin $>2 \mathrm{mg} / \mathrm{dL}$ (HTB). Healthy control was used as the reference. Diagnostic value of total bile acid for CCA was evaluated by ROC analysis. In addition, the correlation between TSBA and liver

${ }^{1}$ Centre for Research and Development of Medical Diagnostic Laboratories, Faculty of Associated Medical Sciences, ${ }^{2}$ Liver Fluke and Cholangiocarcinoma Research Center, Faculty of Medicine, Khon Kaen University, Khon Kaen, Thailand *For correspondence: sirpat@kku.ac.th 


\section{Sutthikan Sombattheera et al}

function parameters were analyzed. The results showed that the TSBA level of CCA patients can be used as the diagnostic marker for CCA patients without jaundice, especially when it was combined with the serum alkaline phosphatase (ALP) level.

\section{Materials and Methods}

\section{Subjects}

Serum samples from 92 patients (57 males and 35 females) with CCA and 46 patients (28 males and 18 females) with benign biliary disease (BBD) who have total serum bilirubin $\leq 2 \mathrm{mg} / \mathrm{dL}$ (low total bilirubin; LTB) were selected at the Liver Fluke and Cholangiocarcinoma Research Center, Srinagarind Hospital, Faculty of Medicine, Khon Kaen University, Thailand. Data of the patients included age, sex and biochemical analysis profile of serum such as cholesterol, albumin, total bilirubin, alanine aminotransferase (ALT), aspartate aminotransferase (AST) and alkaline phosphatase (ALP).

In addition, CCA patients were divided into two categories; those who had total serum bilirubin $\leq 2 \mathrm{mg} / \mathrm{dL}$ (low total bilirubin; LTB) group ( 37 males and 23 females) and those who had total serum bilirubin $>2 \mathrm{mg} / \mathrm{dL}$ (high total bilirubin; HTB) group (20 males and 12 females). Sera were collected also from 115 healthy controls (39 males and 76 females) who visited for health check-up at the office of Medical Technology and Physical Therapy Health Service, Faculty of Associated Medical Sciences, Khon Kaen University, Thailand. Data for control samples also included age, sex and the profile of serum biochemical analysis including cholesterol, ALT, AST and ALP. This study was approved by the Ethics Committee for Human Research (HE561280) of Khon Kaen University.

\section{Total serum bile acids (TSBA) measurement}

Total serum bile acids (TSBA) was determined using the Total Bile Acids Assay kit (Diazyme Laboratories, Poway, CA) based on the enzyme cycling method. Serum sample of $3 \mu \mathrm{L}$ was used for the analysis on the Beckman
Synchron CX4 clinical chemistry analyzer. The results were presented in $\mu \mathrm{mol} / \mathrm{L}$.

\section{Determination of diagnostic value}

The cut-off values were calculated by the receiver operating characteristic (ROC) curve analysis. The percentages of the sensitivity, specificity and accuracy of total bile acids were estimated.

\section{Statistical analysis}

Results were presented as median \pm quartile deviation and the range (minimum to maximum). Data were analyzed using Mann-Whitney U test and Spearman's rank correlation test. The significant level was set at p-value less than 0.05 .

\section{Results}

Correlation between the total serum bile acid level and total bilirubin or other liver function parameters

Total serum bile acid (TSBA) levels (median \pm quartile deviation) of CCA patients with HTB or LTB and that of the control group were $110.6 \pm 122.8,5.6 \pm 3.3$, and $3.9 \pm 1.1$ $\mu \mathrm{mol} / \mathrm{L}$, respectively.

TSBAlevels of overall CCA patients were significantly correlated with total bilirubin (Figure 1A), ALT (Figure 1C), AST (Figure 1D) and ALP (Figure 1E) but not with cholesterol (Figure 1B) and albumin (Figure 1F). When the CCA patients were divided into HTB and LTB groups, TSBA was significantly correlated with total bilirubin (Figure 1A), cholesterol (Figure 1B), and AST (Figure 1D) in HTB group. In LTB group of CCA patients, TSBA levels did not correlate with any one of those tests (Figure 1A-1F, Table 1).

\section{Diagnostic value of TSBA in CCA with LTB}

Since TSBA levels did not correlate with any one of the liver function tests in the CCA with LTB group, we analysed further whether TSBA had a diagnostic value for
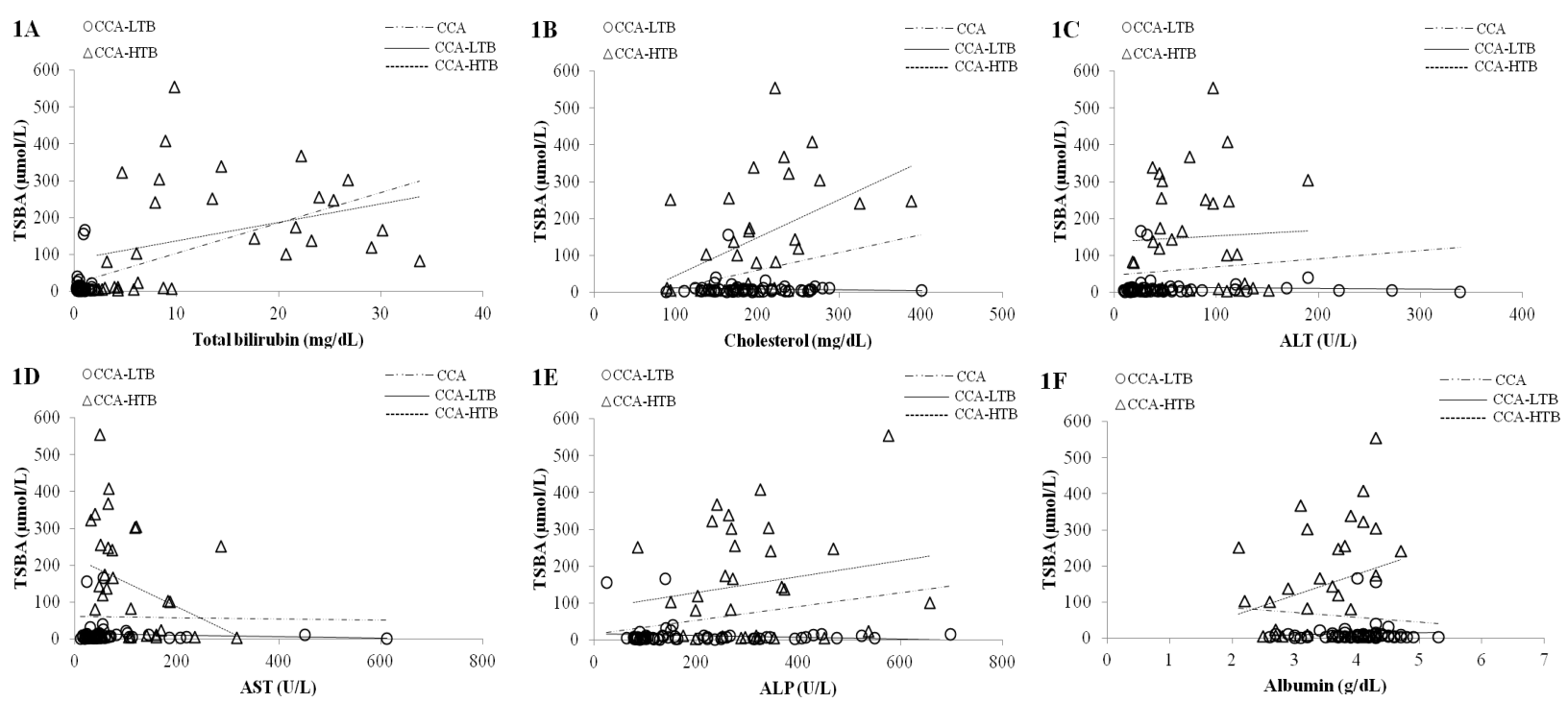

Figure 1. Correlations between Total Serum Bile Acid (TSBA) and Total Bilirubin, Cholesterol, ALT, AST, ALP and Albumin of CCA Patients 
Total Serum Bile Acid as a Potential Marker for the Diagnosis of Cholangiocarcinoma without Jaundice Table 1. Correlation between TSBA Levels and Liver Function Tests in CCA Patients

\begin{tabular}{|c|c|c|c|c|c|c|}
\hline \multirow[b]{2}{*}{ Parameters } & \multicolumn{2}{|c|}{$\mathrm{CCA}$} & \multicolumn{2}{|c|}{ CCA-LTB } & \multicolumn{2}{|c|}{ CCA-HTB } \\
\hline & Correlation coefficient & $\mathrm{p}$-value & Correlation coefficient & $\mathrm{p}$-value & Correlation coefficient & $\mathrm{p}$-value \\
\hline Cholesterol & 0.193 & 0.07 & 0.075 & 0.577 & 0.481 & 0.006 \\
\hline Albumin & -0.121 & 0.252 & 0.044 & 0.737 & 0.212 & 0.243 \\
\hline Total bilirubin & 0.488 & $<0.001$ & -0.135 & 0.303 & 0.594 & $<0.001$ \\
\hline ALT & 0.259 & 0.013 & 0.118 & 0.371 & -0.06 & 0.744 \\
\hline AST & 0.242 & 0.02 & 0.128 & 0.33 & -0.36 & 0.043 \\
\hline ALP & 0.32 & 0.002 & 0.175 & 0.18 & 0.11 & 0.548 \\
\hline
\end{tabular}

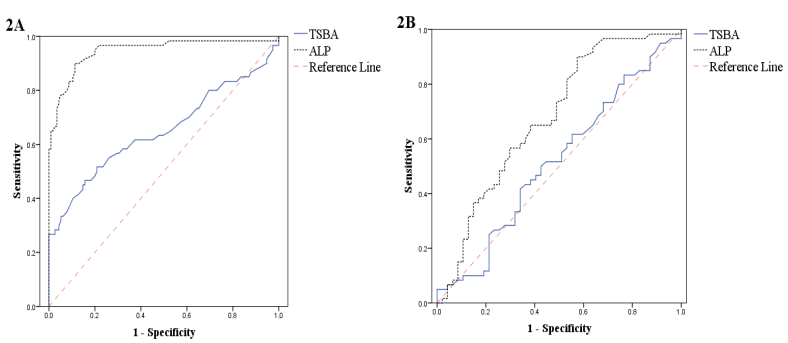

Figure 2. Receiver Operating Characteristic Curve of Total Serum Bile Acid (TSBA) and Alkaline Phosphatase (ALP) for Healthy Control and CCA with LTB (2A), and for BBD with LTB and CCA with LTB (2B)

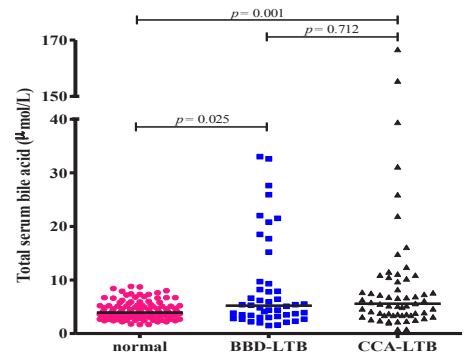

Figure 3. Total Serum Bile Acid (TSBA) in the Normal Group, Benign Biliary Disease with total Bilirubin $\leq 2$ mg/dl (BBD-LTB) and Cholangiocarcinoma Patients with Total Bilirubin $\leq 2 \mathrm{mg} / \mathrm{dl}$ (CCA-LTB)

Table 2. Characteristics of Cholangiocarcinoma Patients with Total Bilirubin $\leq 2 \mathrm{mg} / \mathrm{dL}$ (CCA-LTB)

\begin{tabular}{llll}
\hline Tests & \multicolumn{2}{c}{ TSBA levels of CCA-LTB patients } & p-value \\
\multicolumn{4}{c}{$\begin{array}{c}\text { TSBA } \leq 6.05 \mu \text { mole/L TSBA }>6.05 \mu \text { mole/L } \\
(\mathrm{n}=32)\end{array}$} \\
\hline Age & $61.0 \pm 6.3(38-82)$ & $60.0 \pm 7.6(44-76)$ & 0.331 \\
Cholesterol & $171.0 \pm 29.0(88-263)$ & $188.0 \pm 34.5(123-401)$ & 0.158 \\
Albumin & $4.0 \pm 0.3(2.6-5.3)$ & $4.1 \pm 0.3(2.7-4.7)$ & 0.929 \\
Total bilirubin & $0.7 \pm 0.3(0.2-2.0)$ & $0.4 \pm 0.3(2.2-1.7)$ & 0.221 \\
ALT & $30.0 \pm 15.4(9-339)$ & $35.0 \pm 25.3(12-272)$ & 0.175 \\
AST & $35.0 \pm 11.4(11-612)$ & $49.0 \pm 19.5(15-451)$ & 0.082 \\
ALP & $116.0 \pm 71.5(64-550)$ & $156.0 \pm 115.1(24-698)$ & 0.047 \\
Survival time & $335 \pm 212(30-953)$ & $399 \pm 243(54-1030)$ & 0.296 \\
\hline
\end{tabular}

*Mann-Whitney test Figures represent median \pm quartile deviation (min-max) Normal range: Cholesterol (127-262 mg/dL), Albumin (3.8-5.4 g/dL), Total bilirubin (0.25-1.5 mg/dL), ALT (4-36 U/L), AST (12-32 U/L), ALP (42-121 U/L)

the CCA with LTB group. For this purpose, we performed a ROC curve analysis for the TSBA levels in the sera of the CCA patients with an LTB group and a normal group. The results showed that the cut-off value of the TSBA for the diagnosis of CCA-LTB was $>6.05 \mu \mathrm{mol} / \mathrm{L}$ (Figure $2 \mathrm{~A}$ ) with the sensitivity, specificity and accuracy of $46.7 \%$,

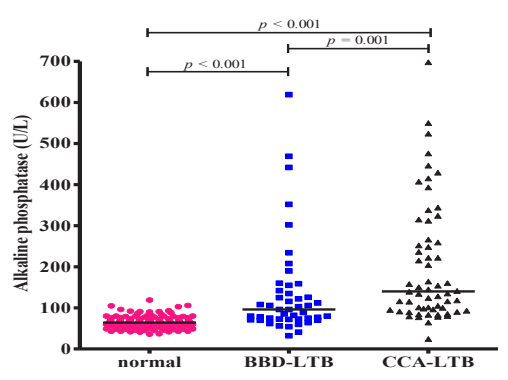

Figure 4. Serum Alkaline Phosphatase in the Normal Group, Benign Biliary Disease with Total Bilirubin $\leq 2$ $\mathrm{mg} / \mathrm{dL}$ (BBD-LTB) and CCA with Total Bilirubin $\leq \mathbf{2}$ mg/dL (CCA-LTB)

$84.4 \%$ and $71.4 \%$, respectively.

When the CCA-LTB group was divided into two subgroups according to TSBA cut-off value of $>6.05$ $\mu \mathrm{mol} / \mathrm{L}$, and various CCA-associated parameters were compared, only ALP was found to be significantly different between two subgroups (Table 2). Based on the significant difference of ALP in two subgroups, we calculated the cut-off value of ALP using ROC curve analysis. The results showed that when cut-off value of ALP was set at $>82 \mathrm{U} / \mathrm{L}$ (Figure 2A), the sensitivity, specificity and accuracy of the diagnostic value of ALP were $90.0 \%, 88.7 \%$ and $89.1 \%$, respectively. When TSBA and ALP were combined as the diagnostic markers, the specificity was increased to $97.4 \%$ as compared with the control value.

Then, we compared the diagnostic efficiency of the TSBA and ALP for CCA with LTB and BBD with LTB. Using ROC curve analysis the cut-off values of the TSBA and ALP were $>6.65 \mu \mathrm{mol} / \mathrm{L}$ and $>82.5 \mathrm{U} / \mathrm{L}$, respectively. When the TSBA and ALP were combined as the diagnostic markers, the specificity increased to $72.9 \%$ compared with TSBA alone (67.4\%) or ALP alone (43.5\%) (Figure 2B).

Since TSBA level seems to have a diagnostic value for CCA with LTB, we compared the TSBA levels in CCA with LTB, benign biliary diseases (BBD) with LTB and control group. The results demonstrated that TSBA level in CCA with LTB was 5.6 \pm 3.3 (median \pm quartile deviation), that in BBD with LTB was 5.2 \pm 3.1 and that in normal control was $3.9 \pm 1.1 \mu \mathrm{mol} / \mathrm{L}$. TSBA in CCA with LTB and BBD with LTB was significantly higher compared with that in normal group (Figure 3). However, TSBA was not statistically different between CCA with LTB and BBD with LTB groups, but CCA demonstrated a trend to be higher. However, ALP in CCA with LTB and BBD with LTB were significantly higher when compared with that for the normal group (Figure 4). 


\section{Discussion}

In CCA with HTB group, the level of TSBA was significantly correlated with the levels of total bilirubin, cholesterol, and AST. In contrast, no significant correlation was observed between TSBA and those liver function tests. This result suggested that the damage of liver cells might not occur in LTB group. The non-jaundiced group had a significantly higher survival rate than the jaundiced group (Sugiyama et al., 1997). The TSBA in CCA with LTB was not correlated with liver function, but more specific to cholangiocytes. As a result, TSBA might be useful as a diagnostic marker for CCA without jaundice.

Bile acid is the end product of cholesterol breakdown, which takes place in hepatocytes. Thus, accumulation of bile acid will occur if there is an impairment in hepatocyte uptake, synthetic or secretion function (Fausa and Gjone, 1976; Shaffer and Gordon, 1978; Tabibian, 1988). In our study, the levels of TSBA in CCA and BBD with LTB were significantly higher than that of the normal group. However, no statistically significant difference was found between CCA and BBD with LTB groups, but CCA has a trend to be higher. Changbumrung et al. (1990) reported that TSBA and conjugated bile acids were elevated in CCA patients. Furthermore, patients with hepatocellular carcinoma also showed significantly higher TSBA than control subjects (Changbumrung et al., 1990; Motawa El- Houseine et al., 2000). However, our results showed that TSBA level alone was not sufficient for discrimination of CCA and BBD.

The concentration of TSBA found in CCA with HTB group was significantly higher than LTB group. Similar to our results, Jusakul et al. (2012) analyzed total bile acids in gallbladder bile, and suggested that the increase in the TSBA levels might be caused by high total bile acids levels in bile and by impaired enterohepatic circulation system (Jusakul et al., 2012).

The serum bile acid concentrations is considered to be more sensitive tests for hepatobiliary diseases than bilirubin, AST and ALT (Fausa and Gjone, 1976). In this study, the liver enzymes such as ALT, AST and ALP in CCA with HTB group was much higher than those in the CCA with LTB group, suggesting that the high total bile acids in HTB group might be caused by liver cell damage (Changbumrung et al., 1990). Determination of TSBA at the cut-off $>6.05 \mu \mathrm{mol} / \mathrm{L}$ gave high specificity but low sensitivity in diagnosis of CCA with LTB patients. When CCA with LTB patients were divided into two subgroups by total bile acids $>6.05 \mu \mathrm{mol} / \mathrm{L}$, ALP was found to be significantly different between the two subgroups. Consequently, we found that the combination of TSBA with ALP increased the specificity compared with the use of TSBA or ALP alone. The persistent elevation of serum ALP is usually the biochemical abnormality leading to the diagnosis of primary sclerosing cholangitis and generally it remains elevated during the course of the disease (Stanich et al., 2011; Al Mamari et al., 2013).

In conclusion, CCA with LTB was found to have significant elevation of TSBA than normal controls. The TSBA level in LTB patients did not correlate with any liver function tests. Nevertheless, the TSBA, in combination with ALP, might be a helpful marker for the detection of CCA suspected patients without jaundice.

\section{Acknowledgements}

We would like to thank the Centre for Research and Development of Medical Diagnostic Laboratories (CMDL), Faculty of Associated Medical Sciences, Khon Kaen University, Khon Kaen, for supporting instruments, and the Liver Flukes and Cholangiocarcinoma Research Center (LFCRC), Faculty of Medicine, Khon Kaen University for kindly providing us serum from CCA patients. We would like to thank Professor Yukifumi Nawa for manuscript editing and the financial support from the Publication Clinic of the Research Affairs, Khon Kaen University.

\section{References}

Al Mamari S, Djordjevic J, Halliday JS, et al (2013). Improvement of serum alkaline phosphatase to $<1.5$ upper limit of normal predicts better outcome and reduced risk of cholangiocarcinoma in primary sclerosing cholangitis. J Hepatol, 58, 329-34.

Bhudhisawasdi V, Khuntikeo N, Chur-in S, et al (2012). Cholangiocarcinoma: experience of Srinagarind hospital. Srinagarind Med J, 27, 331-9.

Blechacz BR, Gores GJ (2008). Cholangiocarcinoma. Clin Liver Dis, 12, 131-50.

Braconi C, Patel T (2010). Cholangiocarcinoma: new insights into disease pathogenesis and biology. Infect Dis Clin North Am, 24, 871-84.

Changbumrung S, Tungtrongchitr R, Migasena P, et al (1990). Serum unconjugated primary and secondary bile acids in patients with cholangiocarcinoma and hepatocellular carcinoma. J Med Assoc Thai, 73, 81-90.

Fausa O, Gjone E (1976). Serum bile acid concentrations in patients with liver disease. Scand J Gastroenterol, 11, 537-43.

Heaton KW (1979). Bile salt tests in clinical practice. Br Med J, 1, 644-6.

Jusakul A, Khuntikeo N, Haigh WG, et al (2012). Identification of biliary bile acids in patients with benign biliary diseases, hepatocellular carcinoma and cholangiocarcinoma. Asian Pac $J$ Cancer Prev, 13, 77-82.

Motawa E. El- Houseine, Mahmoud A. Amer, Abdel Hakim Saad El-Din, et al (2000). Evaluation of serum total bile acids in the diagnosis of hepatocellular carcinoma. J Egypt Natl Cancer Inst, 12, 307-13.

Shaffer EA, Gordon ER (1978). Serum bile acids as related to bile acid secretion in liver disease. Am J Dig Dis, 23, 392-7.

Sripa B, Bethony JM, Sithithaworn P, et al (2011). Opisthorchiasis and Opisthorchis-associated cholangiocarcinoma in Thailand and Laos. Acta Trop, 120, 158-68.

Sripa B, Kaewkes S, Sithithaworn P, et al (2007). Liver fluke induces cholangiocarcinoma. PLoS Med, 4, 201.

Stanich PP, Bjornsson E, Gossard AA, et al (2011). Alkaline phosphatase normalization is associated with better prognosis in primary sclerosing cholangitis. Dig Liver Dis, 43, 309-13.

Sugiyama M, Atomi Y, Kuroda A, et al (1997). Bile duct carcinoma without jaundice: clues to early diagnosis. Hepatogastroenterology, 44, 1477-83.

Tabibian N (1988). Serum bile acid levels in liver disease: is there a clinical application? South Med J, 81, 281-2.

Webster CR, Usechak P, Anwer MS (2002). cAMP inhibits bile acidinduced apoptosis by blocking caspase activation and cytochrome c release. Am J Physiol Gastrointest Liver Physiol, 283, 727-38.

Wiangnon S, Suwanrungruang K, Kamsa-Ard S (2012). Cholangiocarcinoma in Khon Kaen Province. Srinagarind Med $J, 27,326-30$.

Wongkham S, Silsirivanit A (2012). State of serum markers for detection of cholangiocarcinoma. Asian Pac J Cancer Prev, 13 Suppl, 17-27.

Zografos GN, Farfaras A, Zagouri F, et al (2011). Cholangiocarcinoma: principles and current trends. Hepatobiliary Pancreat Dis Int, 10, 10-20. 